\title{
28 Research Square \\ Characterization of Head and Neck Squamous Cell Carcinoma From Jamaican Patients
}

SHARON Rosemarie HARRISON ( $\nabla$ sharon.harrison2@fccc.edu )

Fox Chase Cancer Center https://orcid.org/0000-0002-4764-4538

\section{Camille C Ragin}

Fox Chase Cancer Center

Jeffery Liu

Fox Chase Cancer Center Department of Medical Oncology

\section{Sheray Chin}

University of the West Indies

\section{Marvin Reid}

University of the West Indies

\section{Carlos Escoffery}

University of the West Indies

\section{Short Report}

Keywords: Head and neck cancer, Immunohistochemistry, Polymerase Chain Reaction, Human Papilloma Virus

Posted Date: July 16th, 2020

DOI: https://doi.org/10.21203/rs.3.rs-41709/v1

License: (c) (i) This work is licensed under a Creative Commons Attribution 4.0 International License.

Read Full License 


\section{Abstract}

HNSCC is the sixth most common cancer worldwide and the seventh most common in Jamaica. Mortality rates for HNSCC in the Caribbean are among the highest in the world. Despite this there is no information on the molecular characterization of these cancers and the relative contribution of tobacco and HPV in this population. This study will examine the molecular features of HNSCC as it relates to p16 expression and HPV in patients from Jamaica. There were no previous published studies of this kind conducted in the English-speaking Caribbean.

Methods: This retrospective cohort study was conducted using archival formalin-fixed paraffin-embedded tissue from patients diagnosed with HNSCC at the Department of Pathology, University of the West Indies and the National Public Health Laboratory from 2001-2010. Clinical information was extracted for all patients. P16 immunohistochemistry was performed using P16INK4A clone E6H4 mouse anti-human protein and identification of HR-HPV DNA was done by standard PCR using MY09/MY11 consensus primers of the L1 HR-HPV gene.

Results: The study population consisted of 327 HNSCC cases with ages ranging from 18 to 92 years (mean, 65 yrs. \pm 15 SD). There were 254 males and 63 females (10 unreported sex) with M: F ratio of 4:1. Most tumors were either moderately differentiated $147 / 327$ (45\%) or were poorly differentiated $55 / 327$ (17\%). This study cohort consisted of patients diagnosed with cancer of the larynx (57.5\%), oral cavity (19.3\%), other sites including lip, salivary gland and nasopharynx (14.7\%) and oropharynx (8.6\%). FFPE tissues from all cases were tested for p 16 ; the majority $255 / 327$ (78\%) were negative and $71 / 327(21.7 \%)$ were positive. All p16 + cases along with a subset of 60 randomly selected p16- cases were tested for HRHPV by PCR. There were nine $\mathrm{p} 16$ + cases which were also positive for HR-HPV DNA. HPV 16 was detected in all cases, one case had HPV16 and 18, and another had HPV 16, 18, 39, 68 and 73. None of the sixty p16- cases were positive for HR-HPV. The nine patients that were HR-HPV + and p16 + had a mean age of 61 years \pm 15 SD (range 39-83 years) with a M: F ratio of 8:1. All of these patients had moderately differentiated tumors, from oropharynx and larynx (44.4\%) each and oral cavity $(11.1 \%)$.

Conclusion: In Jamaica, cancer of the larynx is the most common head and neck cancer. The prevalence of HPV-related HNSCC appears to be low with most patients with disease in non-oropharyngeal cancer sites. Based on what is known from the literature, our study results suggest that in Jamaica most patients are diagnosed with alcohol and tobacco driven disease. Interventions to reduce smoking and limit alcohol use are highly relevant in Jamaica. The role of HPV in HNSCC warrants further investigation in this population.

\section{Background}

Head and neck squamous cell carcinomas (HNSCCs) include cancers arising in the mouth/oral cavity, oropharynx, hypopharynx and larynx. HNSCC is the sixth most common cancer worldwide with an estimated annual burden of 358,142 deaths and 705,781 incident cases (1). In Jamaica, HNSCC was the 
fifth most common cancer in Jamaican men from 2008 to 2011 accounting for an age standardized rate of 7.8/100,000 per year. Conversely the disease is relatively uncommon in Jamaican women and accounted for $3.2 / 100,000$ cases per year in the same period (2).

Over the last decade the age of onset, clinical manifestation and the general bio-pathology of HNSCC patients have changed (3). HNSCC can now be divided into two sub-groups: high-risk human papilloma virus (HR-HPV)-associated disease (in the oropharynx) and tobacco-associated disease (in the oral cavity, hypopharynx and larynx), with research demonstrating that patients with HPV-positive disease have a better prognosis in comparison to patients with tobacco- associated disease (4-6). Typically, tobacco associated HNSCC usually manifests with early loss of p16 expression either by hyper-methylation or deletion of the gene (7). In contrast the hallmark of HPV-positive disease is the overexpression of p16. A more recent meta-analysis review reported by Ragin et al 2010, highlighted that there was survival advantage for HPV positive cancer only in patients with oropharyngeal cancer and their risk of death or recurrence was decreased by $28 \%$ and $49 \%$ respectively as opposed to their non- oropharyngeal counterparts. There was, however, a clear disparity in survival between racial ethnic groups with Blacks having poorer outcomes than Whites (8).

There have been no published reports of the HPV status of HNSCC cases in Jamaica and the EnglishSpeaking Caribbean, and we therefore deemed it to be of extreme importance to investigate these tumors for 16 expression and HR-HPV status. This study was a site-specific cohort study using formalin-fixed paraffin- embedded (FFPE) tissues from HNSCC s diagnosed in the Department of Pathology, UWI, and the NPHL over the ten-year period 2001-2010.

\section{Materials And Methods}

\section{Study Material}

Following ethical approval from the University Hospital of the West Indies/University of the West Indies (UHWI/UWI) Ethics Committee, archival FFPE tissue from 327 patients diagnosed with HNSCC at the Department of Pathology, University of the West Indies (DPUWI), and the National Public Health Laboratory (NPHL) between the years 2001-2010 were identified. Original slides used to make the diagnosis were reviewed by the study pathologist to select the best diagnostic tumor tissue available for the assays. A series of 4- $\mu$ m-thick tissue sections were taken from each paraffin block, the first and last slides were stained with hematoxylin and eosin (H\&E) as a quality control (QC), to confirm the diagnosis of SCC, others were used for $\mathrm{p} 16 \mathrm{IHC}$ testing, while others sections were taken before the last QC slide and placed directly into microcentrifuge tubes for DNA extraction and analysis with the usual steps being taken to avoid cross-contamination between cases.

\section{Immunohistochemistry}

P16INK4A CINtec ${ }^{\circledR}$ Histology Kits from MTM Laboratories, INC. Massechausettes, USA was used for this analysis. The standard manual immunohistochemistry methodology was performed following kit 
manufacturer's instructions. CDKN2A (p16) expression was determined by the presence of continuous(diffuse) staining of the basal and parabasal layers of the squamous epithelium observed using a light microscope as defined by CINtec Histology kit insert.

\section{DNA Extraction}

DNA extraction was performed using the Tissue and Hair Extraction Kit (for use with DNA IQ TM) Promega Madison, WI, USA, following manufacturer's instructions. The resulting DNA was stored at $4{ }^{\circ} \mathrm{C}$ for short-term storage or at $-20^{\circ} \mathrm{C}$ or $-70^{\circ} \mathrm{C}$ for long-term storage and used for HPV DNA amplification and genotyping.

\section{HPV Genotyping}

The extracted DNA was amplified using the Innogenetics Gene Amp kit, and HPV genotyping using INNOLiPA HPV Genotyping Extra assay (Fujirebio Inc, GA, USA). The amplicon was stored immediately at $-20^{\circ} \mathrm{C} \pm 5^{\circ} \mathrm{C}$, and then used to perform the reverse line blot hybridization to HPV type-specific immobilized probes for 18 high-risk/ possibly high-risk $(16,18,25,31,33,35,39,45,51,52,53,56,58,59$, $66,68,73,82)$ and 7 low-risk $(6,11,40,43,44,54,70)$ HPV types. In addition to the internal assay control, testing for human DNA was conducted from all specimens (LiPA: the INNO-LiPA HPV prototype research assay). After PCR amplification with biotin-labelled consensus primers, the PCR products were hybridized to specific HPV probes immobilized on a membrane strip. At the end of the process each of the strips was air dried on a clean absorbent paper towel and pasted onto the reporting sheet provided in the kit. Each biotinylated strip contains 28 different oligonucleotide probes including HR, LR and intermediate risk types. A line is considered positive when a clear purple/brown band appears at the end of the test procedure. All clearly visible lines are then scored by using the INNO-LiPA HPV Genotyping Extra Reading Card. This is then compared to INNO-LiPA HPV Genotyping Extra Interpretation Chart supplied with the kit.

\section{Statistical Analysis}

All statistical analyses were done using STATA statistical software, version 12 (College Station TX 77845). Values were expressed as frequencies, proportions, means \pm SD or median with range as appropriate. Differences in proportions were tested with chi square statistics.

\section{Results}

\section{Demographic Characteristics of the HNSCC study population}

The specimens analysed in this study were drawn from two different pathology labs in Kingston, DPUWI and NPHL where the vast majority of all HNSCC cases in Jamaica are diagnosed due to the concentration of Head and Neck Clinical Specialists in this geographic area. Patients diagnosed in these labs and included in this study were from 10/14 Parishes in the entire Island. The description of the study population of HNSCC cases $(n=327)$ is presented in Table 1. The ages at diagnoses were documented 
for $96 \%$ of cases and ranged from 18-92 years (mean, 65yrs. \pm 11 SD) with a 4:1 ratio of males to females. Most patients were diagnosed with laryngeal cancer (57.5\%), oral cavity (19.3\%), other sites including lip salivary gland and nasopharynx $(14.7 \%)$ and $8.6 \%$ of patients were diagnosed with oropharyngeal cancer. Moderately differentiated tumors were the most common overall accounting for $45 \%$ of all tumors analyzed, while the poorly differentiated tumors were the least common accounting for $16.8 \%$.

Table 1

Demographic Characteristics of the HNSCC study population

\begin{tabular}{|c|c|c|c|}
\hline Description & & Frequency & Percent (\%) \\
\hline \multirow[t]{4}{*}{ Age (years) } & $18-60$ & 107 & 32.72 \\
\hline & $61-70$ & 109 & 33.33 \\
\hline & $71+$ & 98 & 29.97 \\
\hline & Unknown & 13 & 3.98 \\
\hline \multirow[t]{3}{*}{ Gender } & Males & 254 & 77.91 \\
\hline & Female & 63 & 19.33 \\
\hline & unknown & 10 & 2.76 \\
\hline \multirow[t]{2}{*}{ Years of diagnosis } & $2001-2005$ & 184 & 56.27 \\
\hline & $2006-2010$ & 143 & 43.73 \\
\hline \multirow[t]{4}{*}{ Anatomical sites } & Larynx & 188 & 57.49 \\
\hline & Oral Cavity & 63 & 19.27 \\
\hline & Oropharynx & 28 & 8.56 \\
\hline & Other & 48 & 14.68 \\
\hline \multirow[t]{4}{*}{ Differentiation } & Well & 108 & 33.03 \\
\hline & Moderate & 147 & 44.95 \\
\hline & Poor & 55 & 16.82 \\
\hline & Unknown/Undifferentiated & 17 & 5.2 \\
\hline
\end{tabular}

\section{P16 Expression}

An example of a positive and negative HNSCC case showing the characteristic p16 staining picture is presented in Fig. 1. Table 2 shows the results for the p $16 \mathrm{IHC}$ testing done of this population. The majority $255 / 327$ (78\%) were negative and $71 / 327$ (21.7\%) were positive for p16 overexpression. The p16 IHC percent positivity was generally low in all subsites except for the oropharynx with $11 / 28$ (39\%). 
Table 2

CDKN2A (p16) expression by Anatomical sites

\begin{tabular}{|lllll|}
\hline Anatomical Sites & $\begin{array}{l}\mathbf{p} 16-\text { positive } \\
\mathbf{N}(\%)\end{array}$ & $\begin{array}{l}\mathbf{p 1 6 - n e g a t i v e} \\
\mathbf{N}(\%)\end{array}$ & $\begin{array}{l}\mathbf{p 1 6 - I n t e r m e d i a t e} \\
\mathbf{N}(\%)\end{array}$ & Total \\
\hline Larynx & $37(20)$ & $148(79)$ & $3(1)$ & $188(100)$ \\
\hline Oral Cavity & $15(24)$ & $46(73)$ & $2(3)$ & $63(100)$ \\
\hline Oropharynx & $11(39)$ & $17(61)$ & $0(0)$ & $28(100)$ \\
\hline Other & $8(17)$ & $38(79)$ & $2(4)$ & $48(100)$ \\
\hline Total & 71 & 249 & 7 & 327 \\
\hline Pearson Chi2(6) $=8.3037 ; \mathrm{Pr}=0.217$ & & \\
\hline
\end{tabular}

\section{HPV Genotyping}

HPV genotyping was performed on 131 samples (Table 3), 71 p16-positives and 60 p16-negatives, the remaining negative samples could not be completed due to reagent shortage. Among these samples 9/131 (7\%) were positive for HPV. HR-HPV type 16 was detected in each of the nine cases and as a single-type infection in all but two cases. The two multiple-type infections carried HR-HPV types 16 and 18 , and $16,18,39,68$ and 73 respectively. The latter patient was the only female and was the youngest patient of the nine (age 39 years), with cancer of the larynx. All the HR-HPV positives cases were detected among the $\mathrm{p} 16$ positive cases. These patients had a mean age of 61 years $\pm 15 \mathrm{SD}$ (range 39-83 years) with a M: F ratio of 8:1. All these patients had moderately differentiated tumors from oropharynx and larynx (44.4\%) each and oral cavity (11.1\%). Table 4 shows the breakdown of HPV/p16 status by sub sites with oropharyngeal cases having the best concordance $4 / 11$ (36\%).

Table 3

HR-HPV PCR positivity among all cases tested by that method

\begin{tabular}{|llll}
\hline Anatomical sites & $\begin{array}{l}\text { HR-HPV-positive } \\
\mathbf{N}(\%)\end{array}$ & $\begin{array}{l}\text { HR-HPV-negative } \\
\mathbf{N}(\%)\end{array}$ & Totals \\
\hline Larynx & $4(5)$ & $71(95)$ & $75(100)$ \\
\hline Oral Cavity & $1(5)$ & $21(95)$ & $22(100)$ \\
\hline Oropharynx & $4(21)$ & $15(79)$ & $19(100)$ \\
\hline Other & $0(0)$ & $15(100)$ & $15(100)$ \\
\hline Totals & 9 & 122 & 131 \\
\hline Pearson chi2(3) $=7.5423 ; \operatorname{Pr}=0.056$ & & \\
\hline
\end{tabular}


Table 4

Comparison of $\mathrm{p} 16$ positive cases with HPV STATUS for

Anatomical sites

\begin{tabular}{|llll|}
\hline Anatomical sites & PCR pos (\%) & PCR neg (\%) & Total N=68 \\
\hline Larynx & $4(11)$ & $32(89)$ & $36(100)$ \\
Oral Cavity & $1(8)$ & $12(92)$ & $13(100)$ \\
Oropharynx & $4(36)$ & $7(64)$ & $11(100)$ \\
Other & $0(0)$ & $8(100)$ & $8(100)$ \\
Totals & 9 & 59 & 68 \\
Pearson chi2(3) $=6.8336 \mathrm{Pr}=0.077$ & \\
\hline
\end{tabular}

\section{Discussion}

This study is the first of its kind in Jamaica and the English-speaking Caribbean and has provided seminal data related to the molecular and epidemiological characteristics of HNSCC patients diagnosed in this population. The mean age of the study population was 65 years. Males with HNSCC patients outnumbered females and this is comparable to worldwide ratios which ranged from a low of 2:1 to a high of 14:1 (9).

There was an overall p16 positivity rate of $22 \%$ and HPV DNA positivity of $7 \%$ with the greatest $\mathrm{p} 16$ positive HPV positive concordance occurring in those from the oropharynx (Table 4). HPV 16 was found in all cases from this population with $77 \%$ of the tumors from the larynx and oral cavity rather than oropharyngeal sites. The overall small number of HPV positives limited the statistical power to generalize, however, the large number of laryngeal cases dictated the conclusion that these findings suggest that in the Jamaican population, the etiological agent driving the disease may be more of the traditional tobacco - associated rather than HPV-associated disease. In contrast, Auguste et al 2017 reported a high prevalence of HR-HPV DNA of $36 \%$ in HNSCC (oral cavity) in a middle aged French West Indies, AfroCaribbean population. The distribution of HPV genotypes 16, 33 and 51, was reported to be more prevalent in HNSCC cases as compared to controls of healthy individuals in the same population (10). It is possible that these differences in HPV prevalence between two Black Caribbean populations may be related to differences in behavioral factors. However further comparative studies are needed to confirm this.

It has been reported that there are racial differences in HPV positivity in patients with HNSCCs in the USA. African-Americans are said to have a lower prevalence of HPV and p16 positivity in their HNSCCs compared to Caucasian-Americans $(11,12)$. HPV 16,18 and CDNK2A (p16) expression was assessed by race, and HPV16,18 positive/ p16 positive was highest among White patients and significantly lower in Asian and Black patients with oropharyngeal when compared to their non- oropharyngeal cancer counterparts. Our results show that cancers of the larynx were the most prevalent in the Jamaican cohort 
which is in line with the current literature in Blacks and there were low numbers of oropharyngeal cancer. There is demonstrable evidence that SCC of the larynx results from lifestyle and environmental factors including heavy cigarette smoking and alcohol abuse (13). There is also emerging information that genetic predisposition may also be playing an important role in the Blacks, as it relates to their ability to metabolize nicotine (14). Health education and promotion activities should therefore address these risk factors to reduce the incidence and impact of HNSCC in this population.

There were some limitations to the study which were all related to the relatively resource poor environment in which the study was conducted. Paraffin block storage was also not always done in the best conditions, which may have affected the ability to extract and amplify HPV DNA. The quantification and verification of quality of double stranded DNA from FFPE tissues is recommended and can be achieved using Nanodrop or Pico green assays. However, this was not feasible due to limited resources and lack of equipment. Therefore, it is likely that many of our DNA samples had a low yield of quality double-stranded DNA. This difficulty was overcome using a highly sensitive HPV assay, the INNO LiPA small fragment PCR technology with the ability to amplify 65 bp fragments Limited resources also resulted in our inability to complete HPV PCR testing for all 327 cases in the study. Regardless, we were able to successfully complete 16 immunohistochemistry on all study samples then strategically test these $\mathrm{p} 16$ positive samples and a random sample of $\mathrm{p} 16$ negatives for HPV DNA. This information provided adequate etiological information about the study population. The documentation of HPV and p16 status in HNSCCs, is currently recommended as an important prognostic indicator for the management of these cancers. It would appear to be judicious that for future work, both the p16 status and HPV type associated in Jamaican patients should be further evaluated in future studies.

\section{Conclusion}

Our study results suggest that in Jamaica most patients are diagnosed with alcohol and tobacco related disease and the need for smoking cessation programs and education may be warranted in this population. In addition, our study identified few patient samples that were HPV positive, but we were limited in HPV testing therefore the role of HPV in HNSCC warrants further investigation in this population.

\section{List Of Abbreviations}

bp Base pairs

DNA Deoxyribonucleic acid

FFPE Formalin Fix Paraffin Embedded Tissue

FMS Faculty of Medical Science

H\&E Hematoxylin \& Eosin Stain 
HNSCC Head and Neck Squamous cell carcinoma

HPV Human Papillomavirus

HR-HPV High-risk HPV

IHC Immunohistochemistry

LiPA Line probe assay

LR-HPV Low-risk HPV

$\mathrm{MOH}$ Ministry of Health

NPHL National Public Health Laboratory

PCR Polymerase chain reaction

SPF short PCR fragment

UHWI University Hospital of the West Indies

UWI University of the West Indies

\section{Declarations}

\section{Ethical approval and consent to participate}

The study was conducted with Ethical approval from the UHWI/UWI/FMS Ethics Committee (ECP245, 2011/2012) and the Director of the NPHL (February 6, 2013),

Consent for publication

Not applicable as study subjects were de-identified, hence anonymous.

Availability of data and materials

The datasets used and/or analyzed during the current are available from corresponding author on reasonable request.

Competing interests

The authors declare that they have no competing interests

Funding 
This study was funded in part by CHASE FUND, CHRC and UWI Graduate studies student Grant to SH. The funding body did not play a role in the design of the study, collection, analysis, and interpretation of data and in writing the manuscript.

\section{Authors contribution}

SH conceptualized the study, performed the laboratory procedures, data analysis, interpretation, contributed to the writing of the manuscript.

CR Principal Investigator and mentor currently, assisted with the preparation of manuscript, proof reading and editing.

JL Assisted with manuscript, proof reading and editing.

SC Co-Investigator and Clinical Oncologist on the study who facilitated the access to patient's information and proof reading.

MR Advisor to the study and assisted with database creation and Statistical analyses.

CE The Principal investigator and mentor for the execution of this graduate research and proof reading.

\section{Acknowledgements}

The authors wish to thankProfessor Darron Brown and BrahimQuadadri from IUPUI, Indianapolis, Indiana, and Professor Wayne McLaughlin and Mr. Compton Beecher and the staff of CARIGEN at the FMS/UWI, Mona Campus for invaluable assistance in PCR analyses.

\section{References}

1. Bray F, Ferlay J, Soerjomataram I, Siegel RL, Torre LA, Jemal A. Global cancer statistics 2018: GLOBOCAN estimates of incidence and mortality worldwide for 36 cancers in 185 countries. CA Cancer J Clin. 2018;68(6):394-424.

2. Bray F, Ferlay J, Laversanne M, Brewster DH, Gombe Mbalawa C, Kohler B, et al. Cancer Incidence in Five Continents: Inclusion criteria, highlights from Volume $\mathrm{X}$ and the global status of cancer registration. Int J Cancer. 2015;137(9):2060-71.

3. Pynnonen MA, Gillespie MB, Roman B, Rosenfeld RM, Tunkel DE, Bontempo L, et al. Clinical Practice Guideline: Evaluation of the Neck Mass in Adults. Otolaryngol Head Neck Surg. 2017;157(2_suppl):1-30.

4. Ang KK, Chen A, Curran WJ Jr, Garden AS, Harari PM, Murphy BA, et al. Head and neck carcinoma in the United States: first comprehensive report of the Longitudinal Oncology Registry of Head and Neck Carcinoma (LORHAN). Cancer. 2012;118(23):5783-92. 
5. Fakhry C, Westra WH, Li S, Cmelak A, Ridge JA, Pinto H, et al. Improved survival of patients with human papillomavirus-positive head and neck squamous cell carcinoma in a prospective clinical trial. J Natl Cancer Inst. 2008;100(4):261-9.

6. Mellin H, Friesland S, Lewensohn R, Dalianis T, Munck-Wikland E. Human papillomavirus (HPV) DNA in tonsillar cancer: clinical correlates, risk of relapse, and survival. Int J Cancer. 2000;89(3):300-4.

7. von Zeidler SV, Miracca EC, Nagai MA, Birman EG. Hypermethylation of the p16 gene in normal oral mucosa of smokers. Int J Mol Med. 2004;14(5):807-11.

8. Ragin CC, Langevin SM, Marzouk M, Grandis J, Taioli E. Determinants of head and neck cancer survival by race. Head Neck. 2011;33(8):1092-8.

9. Simard EP, Torre LA, Jemal A. International trends in head and neck cancer incidence rates: differences by country, sex and anatomic site. Oral Oncol. 2014;50(5):387-403.

10. Auguste A, Gaete S, Herrmann-Storck C, Michineau L, Joachim C, Deloumeaux J, et al. Prevalence of oral HPV infection among healthy individuals and head and neck cancer cases in the French West Indies. Cancer Causes Control. 2017;28(11):1333-40.

11. Stephen JK, Divine G, Chen KM, Chitale D, Havard S, Worsham MJ. Significance of p16 in Sitespecific HPV Positive and HPV Negative Head and Neck Squamous Cell Carcinoma. Cancer Clin Oncol. 2013;2(1):51-61.

12. Weinberger PM, Merkley MA, Khichi SS, Lee JR, Psyrri A, Jackson LL, et al. Human papillomavirusactive head and neck cancer and ethnic health disparities. Laryngoscope. 2010;120(8):1531-7.

13. Gupta B, Johnson NW. Emerging and established global life-style risk factors for cancer of the upper aero-digestive tract. Asian Pac J Cancer Prev. 2014;15(15):5983-91.

14. Blackman E, Ashing K, Gibbs D, Kuo YM, Andrews A, Ramakodi M, et al. The Cancer Prevention Project of Philadelphia: preliminary findings examining diversity among the African diaspora. Ethn Health. 2018:1-17.

\section{Figures}




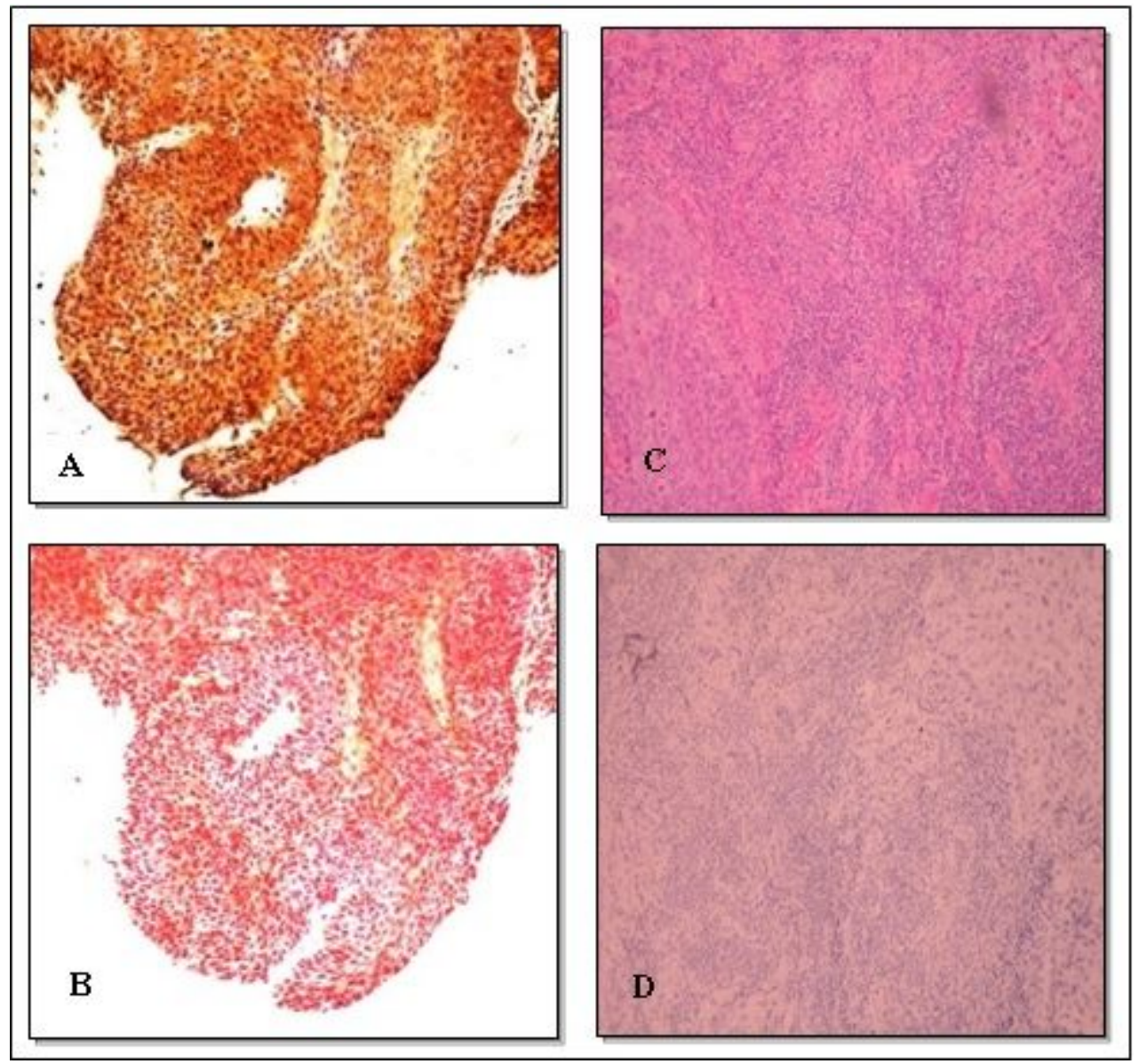

Figure 1

CDKN2A (p16) Staining pattern in HNSCC Cases. (A) p16-positive patient sample with (B)corresponding H\&E slide and (C)corresponding H\&E slide for p16 negative case.(D) a p16-negative patient sample

\section{Supplementary Files}

This is a list of supplementary files associated with this preprint. Click to download.

- CharacterizationofHNSCCfromJamaicanPatients.docx 